\title{
Single Operation to Repair Multifocal Cerebrospinal Fluid Fistulae Following Gunshot Wound: A Case Report
}

\author{
Gabrielle A. White-Dzuro ${ }^{1}$ Pouya Entezami ${ }^{2}$ George Wanna ${ }^{3}$ Paul Russell ${ }^{3}$ Lola B. Chambless ${ }^{1}$ \\ ${ }^{1}$ Department of Neurosurgery, Vanderbilt University, Nashville, \\ Address for correspondence Gabrielle A. White-Dzuro, MD, \\ Tennessee, United States \\ 2 Department of Neurosurgery, Albany Medical Center, Albany, \\ Department of Neurosurgery, Vanderbilt University, Nashville, TN \\ 37235, United States (e-mail: gwhitedz@gmail.com). \\ New York, United States \\ ${ }^{3}$ Department of Otolaryngology, Vanderbilt University, Nashville, \\ Tennessee, United States \\ J Neurol Surg Rep 2016;77:e89-e93.
}

Abstract
Keywords
- cerebrospinal fluid
fistulae
- CSF leak
- endoscopic endonasal
approach
- skull base repair

Introduction Traumatic cerebrospinal fluid (CSF) fistulae can be a challenging neurosurgical disease, often requiring complicated surgical intervention.

Case Presentation A 54-year-old man presented with a gunshot wound to the head with complex injury to the skull base and significant CSF leakage from multiple sites. A single surgery was performed using a combined Neurosurgery, Neurotology, and Rhinology team, which was successful in repairing the multiple skull base defects and preventing further CSF leak.

Discussion Trauma to the skull base is a common inciting factor for the development of CSF fistulae. Endoscopic approaches are often preferred for repairing these defects, but craniotomy remains a viable option that may be required in more complex cases. A combined approach has not been described previously, but was successful for this severe multifocal defect.

Conclusion A multidisciplinary approach allowed for a combined intervention that addressed both the anterior and middle fossae fistulae simultaneously. This limited the potential infectious complications of continued CSF leak and allowed for early rehabilitation.

\section{Introduction}

Cerebrospinal fluid (CSF) fistulae can occur spontaneously as a result of intracranial hypertension, following surgery, or secondary to a traumatic event. This is a common but challenging problem that often requires operative intervention to resolve. Presentation may include rhinorrhea, otorhea, hearing loss, or seizures, with complications that include meningitis, brain abscesses, tension pneumocephalus, and death. ${ }^{1,2}$

Many of the CSF leaks that occur after trauma resolve with conservative measures, such as bed rest, elevation of the head, avoidance of strenuous activities, and decreasing the CSF pressure with spinal taps or drains. ${ }^{3-6}$ A range of surgical interventions are also available for refractory cases, though surgical management requires judicious investigations as it is dependent on the etiology, location, and temporal relationship of the leak with the inciting factor. ${ }^{4-12}$

We present a patient who suffered multiple CSF fistulae following a traumatic gunshot wound and presented with significant CSF rhinorrhea. Instead of staging the procedure and repairing each fistula individually, we used a team of skull base specialists to manage this complex problem with a single surgery to repair all defects simultaneously. received

December 31, 2015 accepted after revision April 18, 2016
DOI http://dx.doi.org/ 10.1055/s-0036-1584281. ISSN 2193-6358. (c) 2016 Georg Thieme Verlag KG
Stuttgart · New York

License terms

$\Theta(1) \Theta \circledast$ 

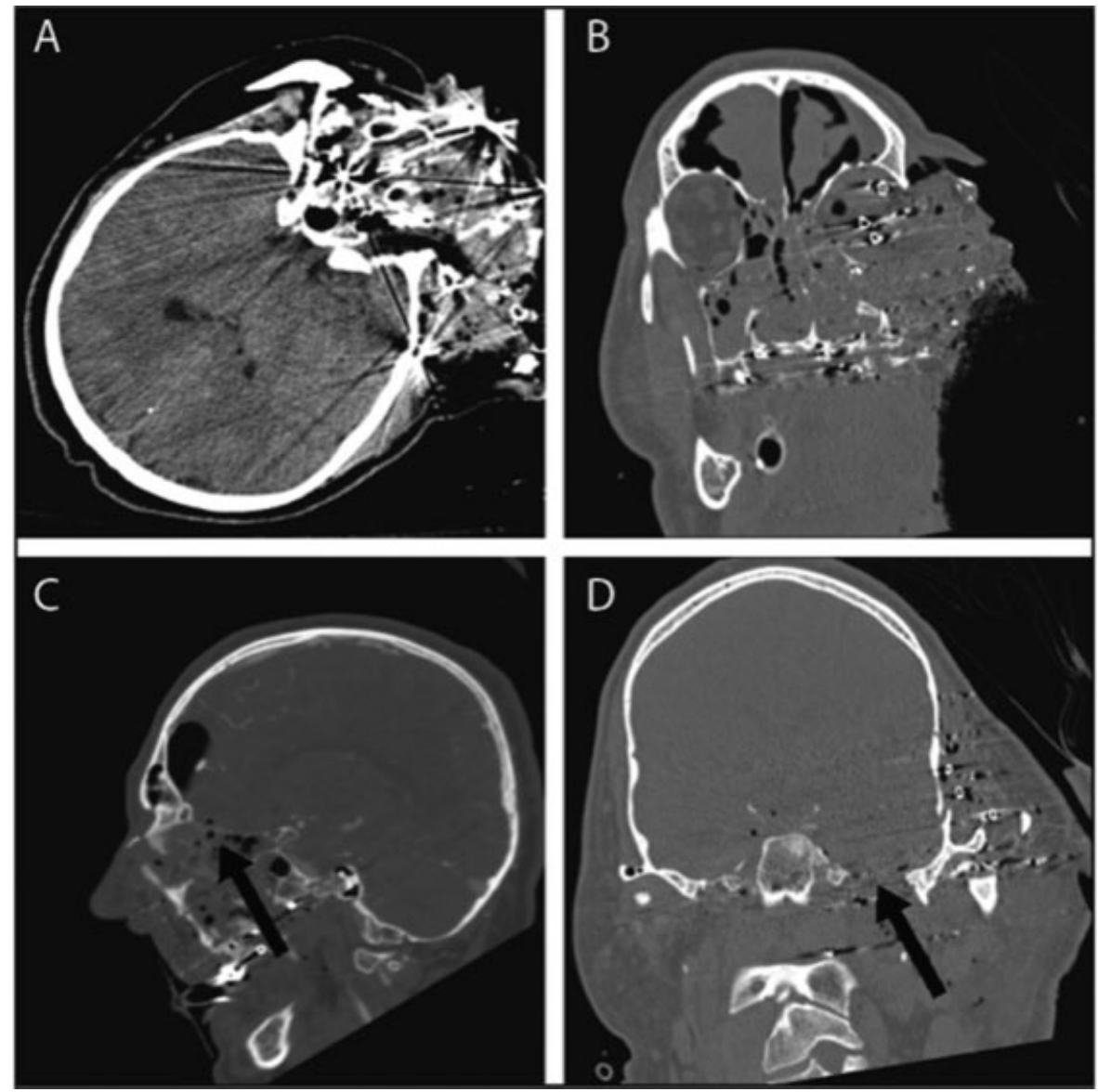

Fig. 1 (A) Noncontrasted sagittal head CT and (B) noncontrasted coronal head CT obtained on admission showing obliteration of the left face, temporal bone, and orbit with a moderate amount of pneumocephalus. (C) Sagittal CT angiography showing anterior encephalocele and destruction of floor of anterior fossa. (D) Coronal head CT obtained on admission showing loss of floor of middle fossa.

\section{Case Presentation}

We present a 54-year-old man with a self-inflicted gunshot wound to the left side of his face and neck. On admission, computerized tomography (CT) scan of his head showed extensive damage to the squamous and petrous portions of the left temporal bone and obliteration of the left orbit and globe. This was associated with a moderate amount of pneumocephalus (-Fig. 1). He was admitted in critical condition and stabilized in the trauma intensive care unit (ICU). He had a significant CSF leak associated with a secondary encephalocele in the anterior and middle fossae. Once it was determined that he had reasonable neurological function and desired repair of his injury, he was taken to the operating room for repair of his extensive skull base fractures. This was done on postinjury day 10 .

A combined Neurosurgical, Neurotological, and Rhinological team planned a modified bicoronal and left-sided middle fossa craniotomy via a single incision with the goal of addressing the anterior and lateral skull base defects simultaneously (-Fig. 2). A lumbar drain was placed after induction with anesthesia for long-term CSF diversion. The first portion of the operation was focused on the anterior skull base and associated encephalocele. An endoscopic endonasal approach allowed the rhinologists to better understand the location of key anatomic landmarks in the setting of extensive fractures. Simultaneously, the neurosurgical team performed a bicoronal craniotomy for direct visualization of the anterior skull base from above. In a combined fashion, the encephalocele was resected and the dura was closed primarily in the anterior skull base. An autologous pericranial flap was placed along the entire defect, and its coverage of the extensive skull base defect was confirmed from the endonasal view.

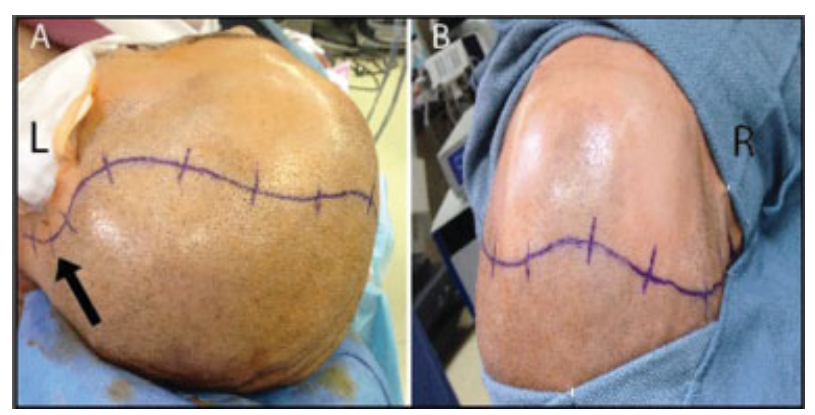

Fig. 2 (A, B) Planned incision with right-sided (R) standard bicoronal incision and left-sided $(\mathrm{L})$ incision modified by retroauricular extension (arrow) to allow a combined frontal craniotomy with middle fossa craniotomy and mastoidectomy. 
Attention was then turned to the left middle fossa. A standard middle fossa craniotomy was performed by the neurosurgical team and a mastoidectomy was performed by the Neurotology team. At that point, the temporal lobe dura was dissected off the skull base. Numerous areas of CSF leak were identified, and it was seen that the intracranial middle fossa was in direct communication with the temporomandibular joint (TMJ). This extensive defect was repaired with a Durepair (Medtronic, Minneapolis, Minnesota, United States) graft. An abdominal fat graft was placed in the tegmen defect around the TMJ, the middle ear, and the mastoid to prevent CSF leak. A multilayer closure of the fascia and skin was undertaken.

Both craniotomy bone flaps were then secured in place using the KLS plating system and the single incision was closed in a layered fashion. Total operating time was 8 hours and 18 minutes, and there was an estimated blood loss of $775 \mathrm{~mL}$. Following the surgical procedure, the patient was transferred to the ICU in stable condition. The patient received $2 \mathrm{~g}$ of Ancef, $1 \mathrm{~g}$ of vancomycin, and $4 \mathrm{~g}$ of cefepime intraoperatively. Vancomycin and cefepime were continued for 7 days postoperatively for meningitis prophylaxis. The lumbar drain was set to drain at $5 \mathrm{~mL} / \mathrm{h}$ postoperatively, and this was continued until it was clamped on postoperative day (POD) 7. The lumbar drain was then removed on POD 8. The patient had a noncontrasted head CT done on POD 1 that showed stable postoperative changes (-Fig. $\mathbf{3}$ ).

The patient convalesced well and was discharged to a long-term acute care facility with the appropriate followup. The patient has subsequently been seen in follow-up for 9 months without any complication. He has had no further CSF leakage or infection and has returned to most of his usual activities.

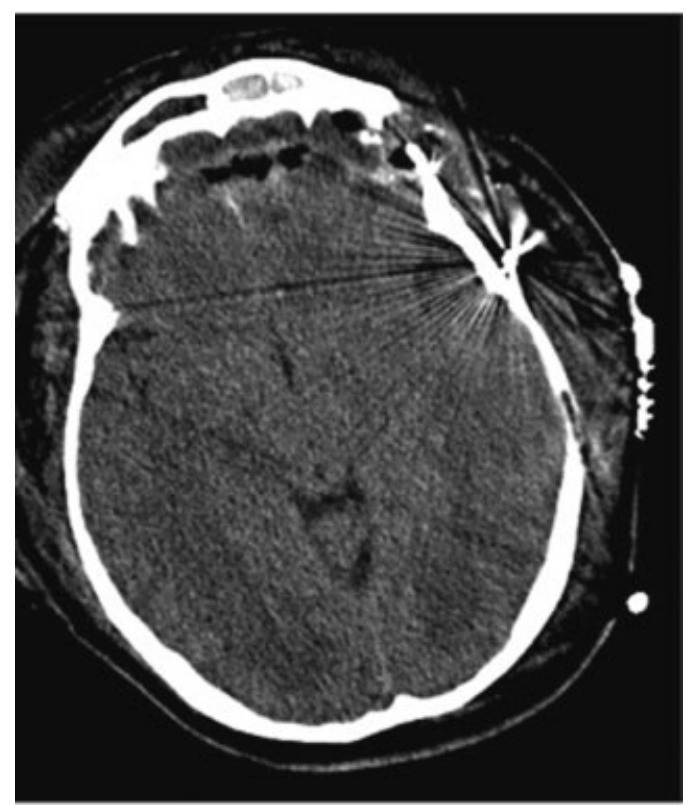

Fig. 3 Noncontrasted axial head CT completed on POD 1 showing stable postoperative changes.

\section{Discussion}

To our knowledge, repair of multifocal CSF fistulae in the setting of gunshot wounds to the head has never been documented in the literature. Our skull base center has extensive experience with the management of CSF fistulae, and we drew upon that experience to plan a novel technical approach to this challenging problem. A team approach utilizing three specialists allowed for a single operation involving a hybrid incision to repair these defects. This included an endoscopic endonasal approach, bicoronal and middle fossa craniotomies, and a mastoidectomy. Our goal was to minimize the risk of infection and allow for early mobilization and rehabilitation by combining these procedures into a single operation.

CSF fistula can be divided based on etiology into traumatic and nontraumatic; trauma accounts for $50.2 \%$, with iatrogenic trauma accounting for $30.1 \%$ of the total amount and accidental trauma accounting for $23.2 \%{ }^{13,14}$ Patients with traumatic anterior skull base deformities commonly present with CSF rhinorrhoea. Dural tears and CSF leaks have been identified in 10 to $30 \%$ of cranial base fractures and are five to six times more frequent in anterior than middle or posterior cranial base fractures. ${ }^{2,9,15-18}$

Historically, frontal or bicoronal craniotomies were the preferred treatment methods, with a success rate between 60 and $90 \%{ }^{5,19}$ To reduce the high failure rate and significant morbidity associated with this approach, minimally invasive endoscopic techniques were attempted. Since Wigand's first documentation of a successful endoscopic CSF leak repair in 1981, the endonasal endoscopic approach has quickly become the preferred surgical approach. ${ }^{20,21}$ While this procedure has a high rate of success and a lower rate of morbidity, there are limitations to what it can treat. In the case of traumatic CSF fistula with multiple fractures of the anterior cranial base, an intracranial approach is preferred. Scholsem and his colleagues described a combined intradural and extradural intracranial approach for repairing traumatic fistulae. $^{6}$ They presented a case series of 209 patients with complex fractures of the anterior skull base with associated CSF fistulae. Of the 209 patients, 109 underwent a bicoronal craniotomy with a combined intradural and extradural approach to identify and repair the CSF fistula. They had a $90 \%$ success rate with the initial procedure, with 11 patients requiring a second-look surgery. Seven patients underwent an endoscopic approach and four patients had a repeat intracranial procedure. Of the patients who underwent surgery, five developed meningitis that was treated with intravenous antibiotics. Conversely, Locatelli et al presented a case series of 135 patients with traumatic CSF rhinorrhea treated through an endoscopic endonasal approach. While they had excellent results with a success rate of $93.3 \%$ on the first attempt, they specified that the key to this approach is an accurate preoperative location of the CSF fistula, an exsanguine operating field, definition of the leak dimensions, and clarification of the subjective anatomy of the area. ${ }^{8}$ This treatment is therefore preferred for circumscribed fractures of the medial portion of the anterior cranial base. ${ }^{7-9,11,12}$ 
In this complex case, we felt our best chance for success involved utilizing both the intracranial and endoscopic approaches simultaneously. Due to the extensive damage to the anterior cranial base, the numerous CSF fistulae, and the associated encephalocele, an intracranial approach was required for effective treatment. The endoscopic approach was added to allow the surgeons to better understand the key anatomic landmarks in the setting of extensive fractures and to ensure that the pericranial graft completely covered the deepest aspect of the bony defect. Similarly, Fishman and his colleagues described similar success in their case series of nine pediatric patients with extensive cranial fractures who underwent a combined intracranial and subcranial approach for repair of posttraumatic CSF leaks. None of the patients that they operated on had meningitis or leak after the combined approach, suggesting that appropriate closure of the dural defect and base of the skull had been achieved. ${ }^{22}$

We utilized a vascularized pericranial flap to repair the anterior skull base defect. We believed that this would allow the region to heal adequately since it achieved excellent coverage of the defect. We also performed a primary repair of the dura of the anterior skull base. While current literature debates the use of lumbar drains with vascularized flaps for repair of skull base defects, ${ }^{23}$ we opted to place a lumbar drain for temporary CSF diversion because of the multiple regions involved and the severity of the injury. In the middle fossa, the dura was shredded in numerous locations and was unable to be repaired primarily. This defect also communicated with the TMJ, leaving a potential space in which a pseudomeningocele could form.

Temporal bone CSF fistulae most commonly occur after surgery to the skull base, traumatic fractures, as a result of erosion from intracranial tumors, or after radiation therapy. $^{24-27} \mathrm{~A}$ large series of traumatic temporal bone fractures found the incidence of CSF leak to be $17 \%{ }^{28}$ Certain principles of management of temporal bone CSF fistulae are widely agreed upon, including the need for surgical repair. The risk of meningitis increases with traumatic leaks that persist beyond 1 week, so surgical intervention should be performed early. ${ }^{28}$

Multifocal CSF fistulae involving both the anterior and middle fossa skull base are rare and occur most commonly following a traumatic event. This case report proposes a unique combined surgical approach to repair all of these defects simultaneously. We believe that in doing so, the potential complications associated with continued CSF leak and communication between the extracranial and intracranial compartments are avoided. In a staged approach, the patient would not only have to undergo two separate rounds of anesthesia, but also be at risk for infection of the newly implanted hardware between the first and second procedure. By combining the procedures, we minimized operating room (OR) time, anesthesia time, and set the patient up to make a quicker recovery. Such an approach requires a team of skull base surgeons with excellent understanding of anterior and middle fossa anatomy as the anatomic distortions present in such a complex case could lead to morbidity if not fully appreciated.

\section{Conclusion}

This case presents a novel operative approach to multifocal CSF fistulae following a gunshot wound. This multidisciplinary approach allowed for a single operation that addressed both his anterior and middle fossae fistulae simultaneously through a single incision. Undergoing a single operation as compared with a staged approach limited the potential complications of continued CSF leak, including meningitis and hardware infection. Despite the severity of his initial injury, this patient recovered well with no perioperative complications.

\section{Conflict of Interest}

The authors declare that they have no conflicts of interest concerning this article.

\section{References}

1 Plein CT, Langerman AJ, Redleaf MI. Bilateral middle cranial fossa encephaloceles presenting as conductive hearing loss. Ear Nose Throat J 2013;92(12):E14-E16

2 Yilmazlar S, Arslan E, Kocaeli $\mathrm{H}$, et al. Cerebrospinal fluid leakage complicating skull base fractures: analysis of 81 cases. Neurosurg Rev 2006;29(1):64-71

3 Dalgic A, Okay HO, Gezici AR, Daglioglu E, Akdag R, Ergungor MF. An effective and less invasive treatment of post-traumatic cerebrospinal fluid fistula: closed lumbar drainage system. Minim Invasive Neurosurg 2008;51(3):154-157

4 Liao W, Lin SH, Huang HT. Traumatic nasal cerebrospinal fluid fistula: report of 86 cases [in Chinese]. J First Mil Med Univ 2003; 23(6):629-630

5 Park JI, Strelzow VV, Friedman WH. Current management of cerebrospinal fluid rhinorrhea. Laryngoscope 1983;93(10):1294-1300

6 Scholsem M, Scholtes F, Collignon F, et al. Surgical management of anterior cranial base fractures with cerebrospinal fluid fistulae: a single-institution experience. Neurosurgery 2008;62(2):463-469, discussion 469-471

7 Daly DT, Lydiatt WM, Ogren FP, Moore GF. Extracranial approaches to the repair of cerebrospinal fluid rhinorrhea. Ear Nose Throat J 1992;71(7):311-313

8 Locatelli D, Rampa F, Acchiardi I, Bignami M, De Bernardi F, Castelnuovo P. Endoscopic endonasal approaches for repair of cerebrospinal fluid leaks: nine-year experience. Neurosurgery 2006;58(4, Suppl 2):ONS-246-ONS-256, ONS-256-ONS-257

9 Loew F, Pertuiset B, Chaumier EE, Jaksche H. Traumatic, spontaneous and postoperative CSF rhinorrhea. Adv Tech Stand Neurosurg 1984;11:169-207

10 Rocchi G, Caroli E, Belli E, Salvati M, Cimatti M, Delfini R. Severe craniofacial fractures with frontobasal involvement and cerebrospinal fluid fistula: indications for surgical repair. Surg Neurol 2005;63(6):559-563, discussion 563-564

11 Zweig JL, Carrau RL, Celin SE, et al. Endoscopic repair of cerebrospinal fluid leaks to the sinonasal tract: predictors of success. Otolaryngol Head Neck Surg 2000;123(3):195-201

12 Nohra G, Jabbour P, Haddad A, Abouhamad W, Abilahoud G, Okais N. Subcranial subfrontal approach for the treatment of extensive cerebrospinal fluid leaks [in French]. Neurochirurgie 2002;48(2-3, Pt 1):87-91

13 Har-El G. What is "spontaneous" cerebrospinal fluid rhinorrhea? Classification of cerebrospinal fluid leaks. Ann Otol Rhinol Laryngol 1999;108(4):323-326 
14 Psaltis AJ, Schlosser RJ, Banks CA, Yawn J, Soler ZM. A systematic review of the endoscopic repair of cerebrospinal fluid leaks. Otolaryngol Head Neck Surg 2012;147(2):196-203

15 Lewin W. Cerebrospinal fluid rhinorrhea in nonmissile head injuries. Clin Neurosurg 1964;12:237-252

16 Marentette LJ, Valentino J. Traumatic anterior fossa cerebrospinal fluid fistulae and craniofacial considerations. Otolaryngol Clin North Am 1991;24(1):151-163

17 Meco C, Oberascher G. Comprehensive algorithm for skull base dural lesion and cerebrospinal fluid fistula diagnosis. Laryngoscope 2004;114(6):991-999

18 Piek J. Surgical treatment of complex traumatic frontobasal lesions: personal experience in 74 patients. Neurosurg Focus 2000;9(1):e2

19 Costa $\mathrm{H}$, Cerejo A, Baptista A, et al. The galea frontalis myofascial flap in anterior fossa CSF leaks. Br J Plast Surg 1993;46(6):503-507

20 Savva A, Taylor MJ, Beatty CW. Management of cerebrospinal fluid leaks involving the temporal bone: report on 92 patients. Laryngoscope 2003;113(1):50-56

21 Wigand ME. Enlarged middle fossa approach to the cerebellopontine angle. Technique and indications [in French]. Rev Laryngol Otol Rhinol (Bord) 1998;119(3):159-162
22 Fishman G, Fliss DM, Benjamin S, et al. Multidisciplinary surgical approach for cerebrospinal fluid leak in children with complex head trauma. Childs Nerv Syst 2009;25(8): 915-923

23 Stokken J, Recinos PF, Woodard T, Sindwani R. The utility of lumbar drains in modern endoscopic skull base surgery. Curr Opin Otolaryngol Head Neck Surg 2015;23(1):78-82

24 Glasscock ME III, Dickins JR, Jackson CG, Wiet RJ, Feenstra L. Surgical management of brain tissue herniation into the middle ear and mastoid. Laryngoscope 1979;89(11):1743-1754

25 Lalwani AK, Jackler RK, Harsh GR IV, Butt FY. Bilateral temporal bone encephaloceles after cranial irradiation. Case report. J Neurosurg 1993;79(4):596-599

26 Mosnier I, Fiky LE, Shahidi A, Sterkers O. Brain herniation and chronic otitis media: diagnosis and surgical management. Clin Otolaryngol Allied Sci 2000;25(5):385-391

27 Neely JG, Kuhn JR. Diagnosis and treatment of iatrogenic cerebrospinal fluid leak and brain herniation during or following mastoidectomy. Laryngoscope 1985;95(11):1299-1300

28 Brodie HA, Thompson TC. Management of complications from 820 temporal bone fractures. Am J Otol 1997;18(2):188-197 